\title{
Pre-clinical dosimetry of a new six-channel applicator for high-dose-rate treatment of esophageal cancer
}

\author{
Anzi Zhao, MS', Shengaiang Gao, PhD², John Greskovich, MD', Douglas Allan Wilkinson, PhD' \\ 'Department of Radiation Oncology, Cleveland Clinic, Cleveland, USA, ${ }^{2}$ Lerner Research Institute, Cleveland Clinic, Cleveland, USA
}

\begin{abstract}
Purpose: To evaluate the dosimetry of a six-channel high-dose-rate (HDR) applicator for treatment of esophageal cancer with respect to lateral directionality and heterogeneous media.

Material and methods: A computed tomography (CT)- and magnetic resonance imaging (MRI)-compatible esophageal applicator consisting of 2 inflatable portions (anchor and therapeutic balloons) with 6 longitudinal treatment catheters equally spaced circumferentially was constructed. Treatment plans were prepared using Oncentra 4.5 for various catheter loadings and target locations and sizes. Calculated dose distributions were compared to measured distributions obtained using film and a water phantom. Balloon inflations with water and with air were tested.

Results: TG-43 dose calculations matched measurements well when inflation balloons were filled with water. When air was used to inflate, model-based dose calculations (TG-186) improved the comparison with measurement. Several cases with simulated ring targets demonstrated better dose conformity to non-uniform targets compared to a single central catheter. Additionally, the use of this applicator compared to a single catheter, gave rise to considerable improvement in sparing non-target tissue.

Conclusions: Lateral dose modulation is achievable with the applicator described in this work. The use of TG-186 dose calculation made a small improvement in heterogeneous media.

Key words: esophageal cancer, HDR applicator, TG-186, dosimetry.

\section{Purpose}

Esophageal cancer is one of the fastest growing and deadliest cancers in the world. During the past three decades, cancers originating in the lower esophagus have increased by $600 \%$ [1]. In Western countries, it is this adenocarcinoma form that develops in the distal esophagus that primarily accounts for the increase [2]. In 2018, the estimated incidence of esophageal cancer in the United States was 17,290 cases, and the estimated number of deaths was 15,850 [3]. Worldwide, esophageal cancer is the $8^{\text {th }}$ most common cancer, with an estimated 572,034 new cases and 508,585 deaths predicted in 2018, making it the world's $6^{\text {th }}$ deadliest cancer [4]. Earlier diagnosis and innovative therapies for esophageal cancer treatment are dearly needed.

For esophageal cancer patients treated with a non-operative approach, phase III trials using concurrent chemoradiation therapy with external beam radiation doses of 50-64 Gy have revealed suboptimal tumor control with high, crude local failure rates of $45-55 \%[5,6]$. Further dose escalation with external beam radiation therapy to improve these failure rates seems improbable since the nearby, radiosensitive organs at risk, such as the lungs, heart, trachea, major bronchi, and spinal cord, limit the amount of external radiation that can be delivered safely.

Intraluminal high-dose-rate (HDR) brachytherapy is an efficient method of delivering high doses to esophageal tumor with adequate spatial precision as a boost after external beam, or as a primary therapy for early-stage, localized esophageal cancer [7, 8]. According to the American Brachytherapy Society (ABS) consensus guidelines for brachytherapy of esophageal cancer, relative to external beam radiation therapy (EBRT), brachytherapy offers the potential advantage of increasing the dose to esophageal tumor while delivering a relatively low-dose to the surrounding normal tissues, particularly the lung, spinal cord, and adjacent normal esophageal mucosa [9]. However, concerns have been raised that the rapid dose fall-off seen in a standard single-channel intraluminal technique has disadvantages, as the dose delivered to the mucosal surface is much higher than that delivered to the prescription depth [10] as well as that brachytherapy may
Address for correspondence: Douglas Allan Wilkinson, PhD, Department of Radiation Oncology, Cleveland Clinic, 9500 Euclid Avenue, Cleveland, OH 44195, USA, phone: +1-216-445-8289,

e-mail: wilkina@ccf.org
Received: 17.07 .2020

Accepted: 10.03.2021

Published: 12.05.2021 
lead to an unacceptable incidence of acute and chronic esophageal ulcers, fistulas, or stricture formation, particularly when combined with concurrent chemotherapy and EBRT [11].

Institutions have investigated HDR brachytherapy in early-stage esophageal cancer patients as a boost after external beam radiation or as a primary therapy. Tamaki and colleagues demonstrated a local control rate of $79 \%$ at 5 years for 54 patients undergoing external beam radiation therapy, followed by 2 or 3 fractions of brachytherapy [12]. A prospective, phase I/II Radiation Therapy Oncology Group (RTOG) trial was completed using five weeks of external beam radiation with chemotherapy, followed by three fractions of HDR brachytherapy [11]. Unfortunately, a high-rate of treatment toxicity, including a 1-year actuarial fistula rate of $18 \%$, was reported using a standard, single brachytherapy catheter technique. Two recent reviews have examined the clinical literature on the role of brachytherapy in early-stage esophageal cancer [13] and in palliative treatment for esophageal cancer [14]. In the first review, twelve studies involving 514 patients with stage 1 cancer were analyzed for local control, disease-free survival, and overall survival, following brachytherapy (low-dose-rate [LDR] and HDR) with or without external beam therapy. They concluded that the combination therapy was effective enough to be considered an alternative to surgery. In the other review, seven randomized studies with 905 patients were included. The authors concluded that brachytherapy improved both dysphagia-free survival and quality of life compared to other treatments (external beam radiation, photodynamic therapy, stenting, laser, and argon plasma coagulation). The most recent data in these reviews showed quite variable toxicity, ranging up to $23 \%$.

Esophageal HDR treatments have typically been delivered using a single-catheter with the dose prescribed to a depth of $5-10 \mathrm{~mm}$. Because of the uncertainty of the position of a single brachytherapy catheter relative to the esophageal tumor target volume, we had previously developed a novel three-catheter technique that improved the dose homogeneity and normal tissue maximum doses [15]. We have now improved our esophageal brachytherapy applicator design by inventing a balloon repositioning, multichannel applicator using 5 or 6 peripheral channels that extend along the length of the applicator over a therapeutic balloon, and terminate at the midpoint of an anchor balloon. Our goal is to optimize dose coverage for a three-dimensional tumor target by modulating the brachytherapy dose within these 5 or 6 channels. The therapeutic and distal anchor balloons of this applicator may be filled with water or air to displace the channels up against the esophageal wall/tumor.

In this study, we evaluated the three-dimensional radiation dose distribution of this new multi-channel esophageal applicator prototype with either air or water filled therapeutic balloon. Additionally, we investigated improvements in three-dimensional dose mapping by increasing the number of channels available for three-dimensional planning. We theorize that this new applicator will allow better conformality of dose to the target, and lower doses within the healthy, normal tissues, poten- tially allowing for safe brachytherapy dose escalation. The studies presented here were done on a prototype, which we envision will lead to clinical use and possibly, a multi-center trial.

Dose calculations for ${ }^{192}$ Ir HDR brachytherapy typically assume a water equivalent medium. However, it has been shown that for HDR treatments of the esophagus, ignoring the effects of nearby bony structures and air in the trachea, can lead to a $15 \%$ error in dose estimation in various organs at risk [16]. Since, the applicator described in this work will most likely be inflated with air and not water due to weight considerations, we have used a model-based computations available in Oncentra TPS to evaluate the effect of that heterogeneity.

\section{Material and methods}

A new inflatable, repositionable, multi-channel applicator for esophageal HDR brachytherapy has been developed in the polymer core laboratory in collaboration with the Cleveland Clinic Medical Device Solutions Team. The applicator has two inflatable balloons: a spherical, anchor balloon located just proximal to a semi-hard distal tip, and a cylindrical, therapeutic balloon located proximal to the anchor balloon. The balloons are made of silicone and nylon. On the periphery of the therapeutic balloon are six LumenCare ${ }^{\circledR}$ applicators (Elekta AB, Stockholm, Sweden). The applicator is both CT and MRI compatible. Both the anchor and therapeutic balloons can be inflated with water or air. For the film dosimetry measurements, $80 \mathrm{ml}$ of air or $50 \mathrm{ml}$ of water were introduced into the therapeutic balloon, respectively, using a standard syringe. A simple water phantom was constructed using a rectangular plastic bin filled with 45 liters of distilled water, and containing several slabs of solid water, onto which film and the applicator could be taped. Figure $1 \mathrm{~A}$ is a photo of the water phantom with the multi-channel applicator taped to a slab of solid. Figure 1B shows two computed tomography (CT) cross-sections of the applicator, one water-filled and the other air-filled. Channels were loaded with guide wires constructed with a train of dummy seeds in order to be identified in CT images. Various channel loadings were used to demonstrate lateral directionality and dose modulation possibilities of this applicator. CT image sets were obtained with our clinical scanner (Phillips Big Bore ${ }^{\circledR}$, Philips North America Corporation, Andover, MA, USA). CT-based planning was performed with the Oncentra (Elekta) treatment planning system (TPS) v. 4.5, based on TG43 formalism. For TG-186 calculations [17], air in the therapeutic balloon was manually contoured and set to its default density.

For the film data reported here, 400 cGy was prescribed to dose points, $1 \mathrm{~cm}$ away from the applicator surface. Dose was delivered from all 6 channels using MicroSelectron v. 3 (Elekta AB, Stockholm, Sweden) afterloader. A piece of Gafchromic EBT3 film (Ashland Advanced Materials, Bridgewater, NJ, USA) was placed at the prescribed plane as well as directly next to the applicator, respectively, in order to measure planar dose distributions. Films were scanned at least 24 hours later on an Epson Perfection 4990 flatbed scanner (Epson America, 


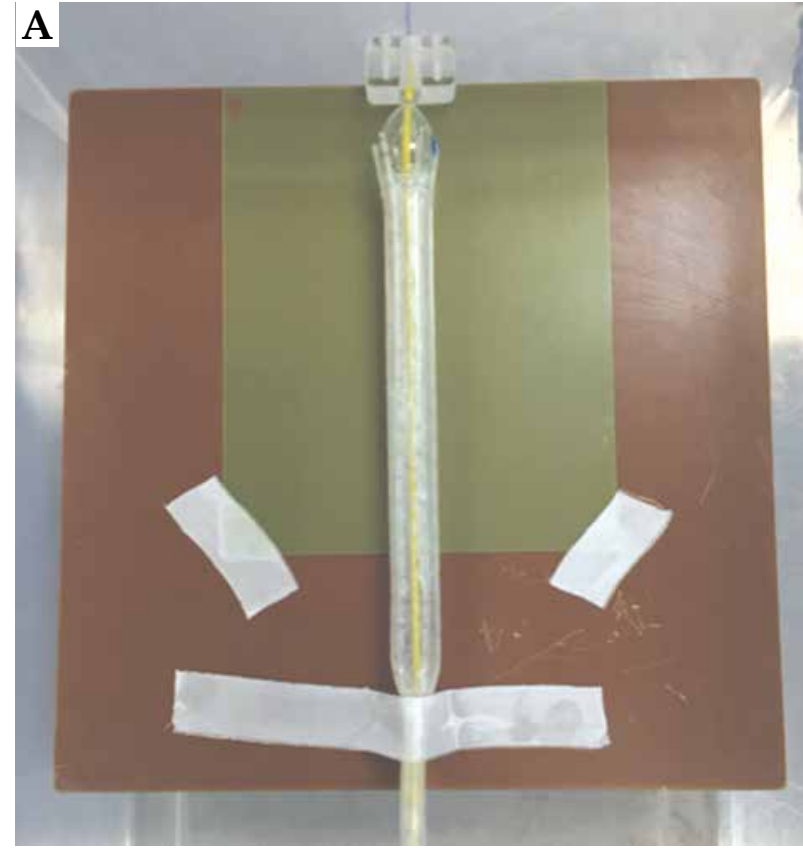

Inc., Long Beach, CA, USA); a film calibration curve was established by exposing film to 15 different doses in the range of 20 cGy to 1000 cGy from a TG-51 calibrated $6 \mathrm{MV}$ linac. Film and TPS generated dose distributions were analyzed using IBA's OmniPro-I'mRT software (IBA USA, Reston, VA, USA). Scanned transverse dose profiles were compared (measured vs. Oncentra calculated), and gamma analysis was performed with a $3 \% / 3 \mathrm{~mm}$ criterion.

Dose modulation was examined using several simulated treatment cases designed to include a range of what might be found in clinical practice: $5 \mathrm{~mm}$ thick ring target
B

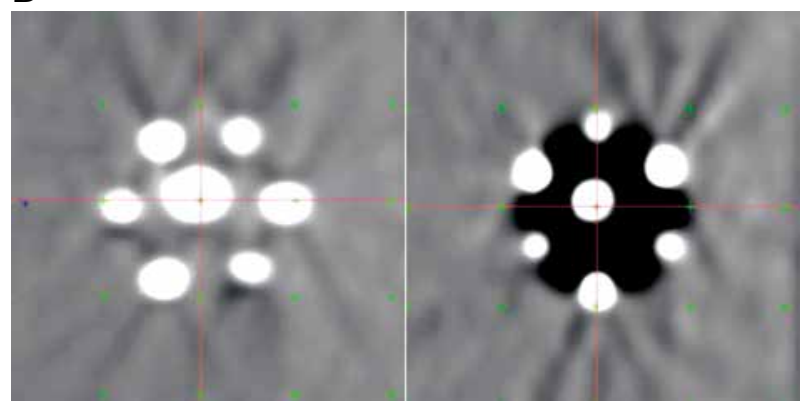

Fig. 1. A) Photograph from above the water phantom with the applicator and film in place; B) CT images of the applicator with a water-inflated (left) and an air-inflated (right) treatment balloon

around the applicator, a $5 \mathrm{~mm}$ thick target consisting of approximately $25 \%$ of the ring circumference, and a ring target of two thicknesses ( 5 and $2 \mathrm{~mm}$ ) of approximately $50 \%$ of the ring circumference. Oncentra image registration tools were used to construct the targets. For all three examples, treatment plans were done to compare dose distributions between utilizing 6 or 3 peripheral channels and a simulated single central channel. Graphical optimization was used to shape the dose distribution for multi-catheter plans. Note that this device does not currently have a single central channel; therefore, the central
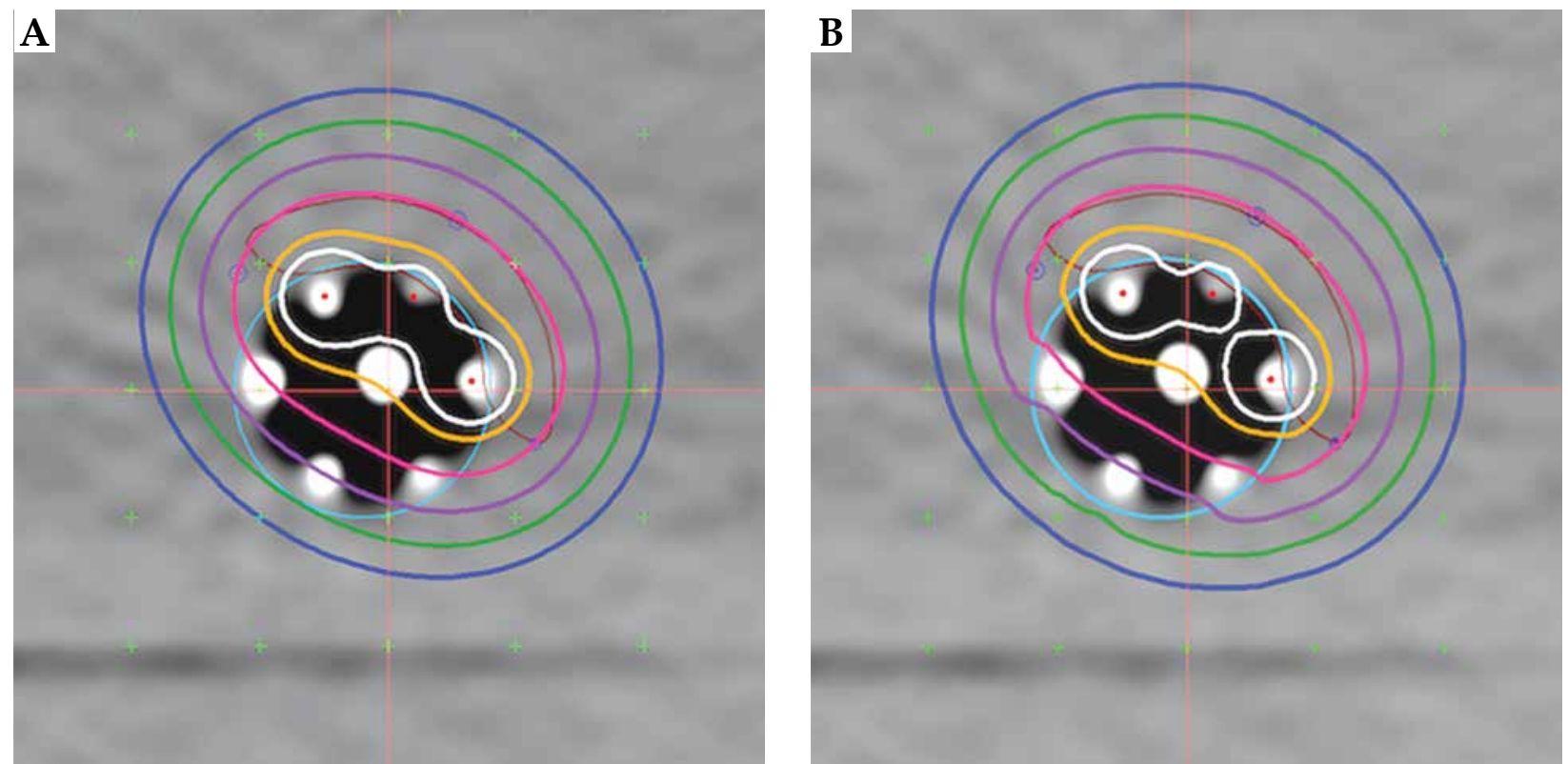

Fig. 2. Comparison of dose distributions in a central cross-sectional plane for three peripheral catheters. A) TG-43 calculated dose (left); B) TG-186 calculated dose (right). The target is in brown; isolines shown are $200 \%$ (white), $150 \%$ (orange), $100 \%$ (red), $75 \%$ (purple), $60 \%$ (green), and 50\% (blue) 

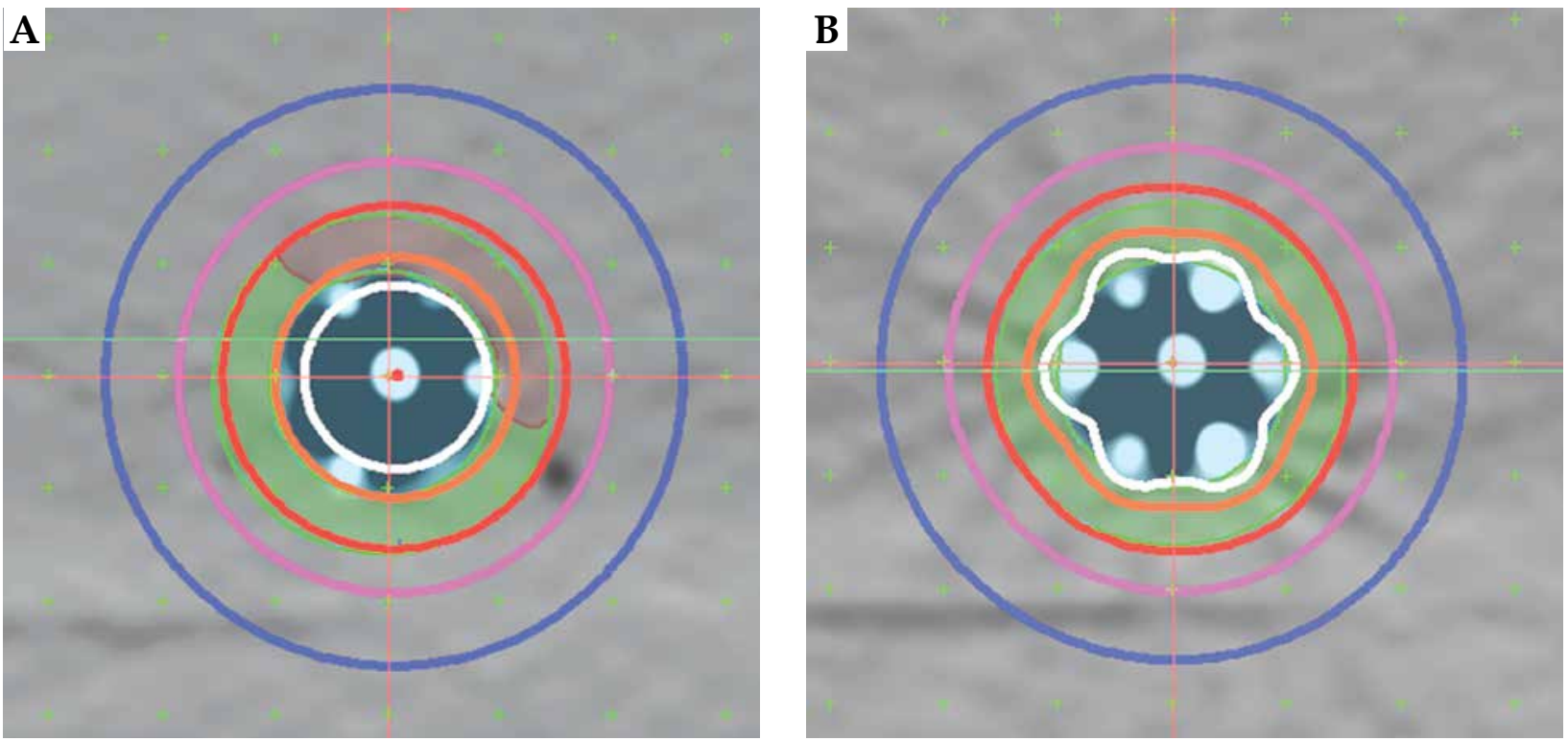

Fig. 3. Central cross-sectional planar dose distributions. A) Single central catheter with dose normalized to $5 \mathrm{~mm}$ from applicator surface; B) 6 catheters used for a circumferential target of $5 \mathrm{~mm}$ thickness. Isolines shown are as in Figure 2 (no $60 \%$ )

rod was digitized as if it were a usable source channel. Dose distributions shown in Figures 2A and 3A, B were calculated using the TG-43 formalism. Dose volume histograms were obtained using default settings in the treatment planning system.

\section{Results}

\section{TG-43 and TG-186 dose distribution}

For commissioning of the TG-186 algorithm (ACE), three test cases from the Brachytherapy Source Registry (IROC, Houston, TX, USA) containing air and water were downloaded and the dose points calculated to be within an average of $1.8 \%$ of expected values [18].

A dose distribution comparison between the TG-43 Oncentra-generated and the film-measured distribution for a coronal plane at the applicator surface is shown in Figure 4. Results for a water-inflated applicator are presented in Figure 4A, using a dose grid of $1 \mathrm{~mm}$. A dose profile comparison in $\mathrm{X}$ direction and a gamma plot for the data were included. There was a small difference at the peak value; gamma analysis resulted in $99.84 \%$ of the pixels passing the $3 \% / 3 \mathrm{~mm}$ criterion. When air was used for inflation in place of water, the TG-43 calculated and measured dose distributions did not agree as well (Figure $4 \mathrm{~B}$ ). Gamma analysis had only $80.8 \%$ of pixels passing the same criterion as above. The dose difference at the peak value was also increased.

When TG-186 calculations were used, the Oncentra and film results were much more similar for the air-filled therapeutic balloon (Figure 4C). The gamma analysis had $99.53 \%$ of pixels meeting the $3 \% / 3 \mathrm{~mm}$ criterion. The peak dose values were very similar.

Dose comparisons were also made between TG-43 and TG-186 for a non-circumferential target $(25 \%)$ de- scribed above. The axial distribution in a plane approximately halfway down the target using TG-43 is shown in Figure 2A; for TG-186, it is in Figure 2B. They appear to be very similar. Dose volume analysis confirmed the similarity. Implant volumes in the range from $V_{50}$ to $V_{200}$ were within $2 \%$ of each other in TG- 43 vs. TG- 186 comparisons. TG-43 values were consistently slightly higher. The contralateral surface had a dose difference of about $3 \%$ (60\% vs. $62 \%$ of prescription). Dose distributions in the next section are for TG-43-based calculations.

\section{Single-centered catheter versus multiple peripheral catheters}

Figure 3 shows the cross-sectional dose distributions for a target ring of $5 \mathrm{~mm}$ thickness, using one central channel (A) or 6 peripheral channels (B). The dose was normalized to $5 \mathrm{~mm}$ depth from the applicator surface; source dwell points were separated by $5 \mathrm{~mm}$ in each channel, using optimization on dose points. Figure 5 is an example of using all 6 channels for a target having 2 thicknesses. Graphical optimization was applied to conform the $100 \%$ isodose line to the target depths. If a single central catheter was used, the dose distribution was as shown in Figure 3A for any of the cases discussed. The volume data provided in Table 1 were taken from dose-volume histogram (DVH) analysis from Oncentra software. The non-target $\mathrm{V}_{100}$ was the volume of $5 \mathrm{~mm}$ ring enclosed by the $100 \%$ isodose that was not a part of the target. It was obtained by subtraction of $\mathrm{V}_{100}$ values from the ring volume $(16.6 \mathrm{cc})$. These data were used to calculate a conformity index (CI = TV/PTV; ICRU 62), for which the treatment volume (TV) was the volume of tissue re-ceiving $100 \%$ dose (it obviously excludes the applicator) and the contoured target was taken as the planning target volume (PTV). 


\section{A}

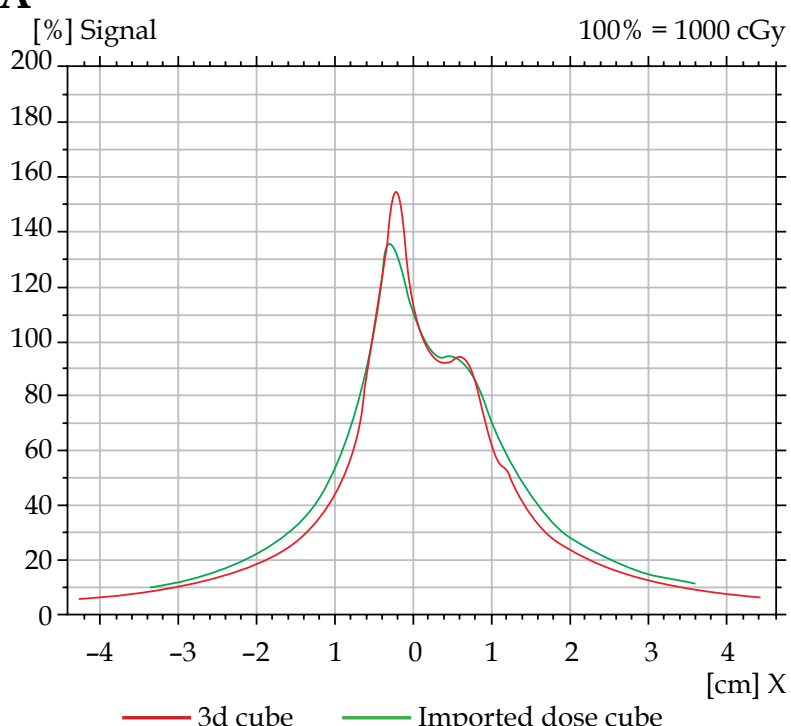

B

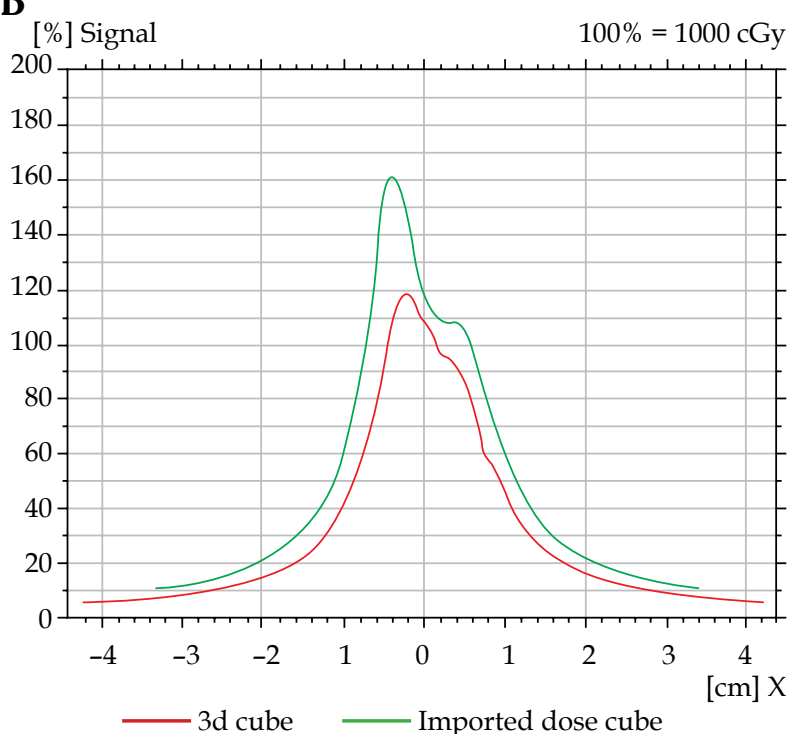

\section{C}

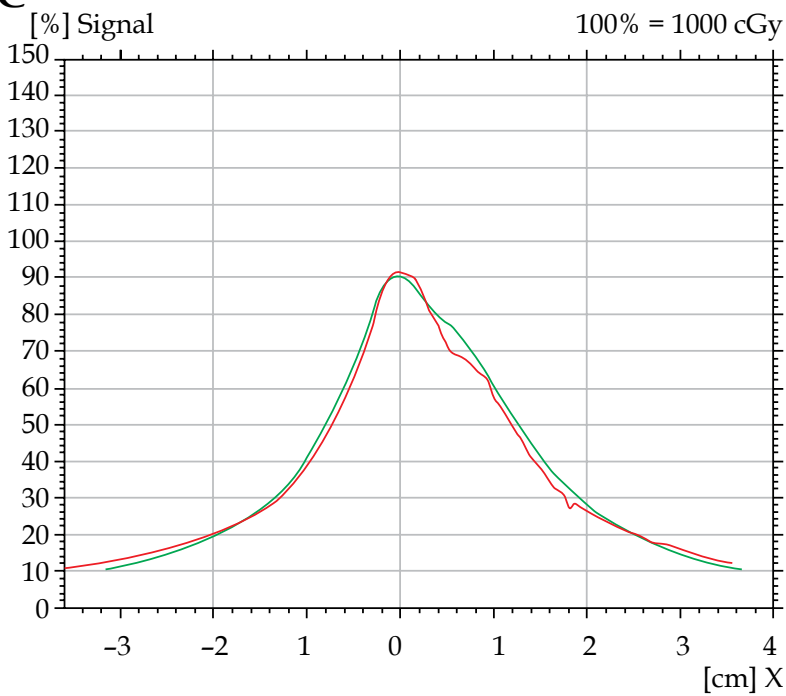

- $3 \mathrm{~d}$ cube $\quad$ Imported dose cube

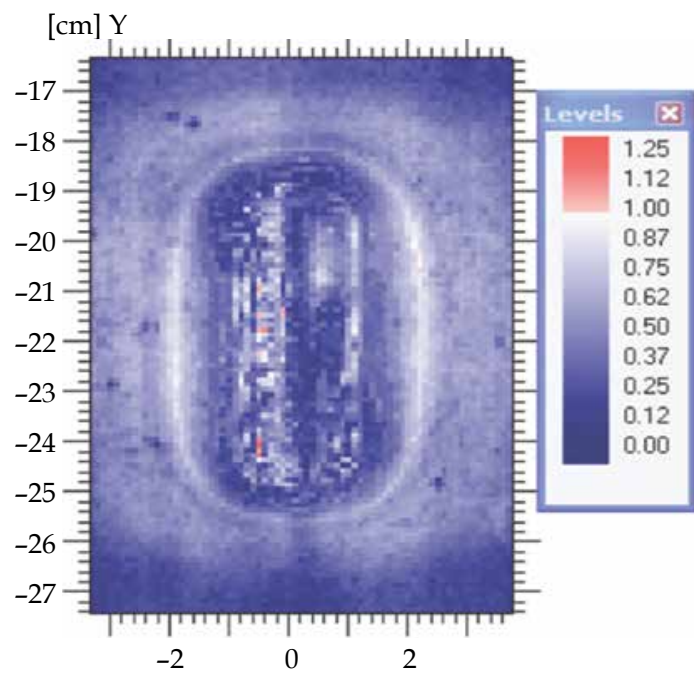

$[\mathrm{cm}] \mathrm{Y}$

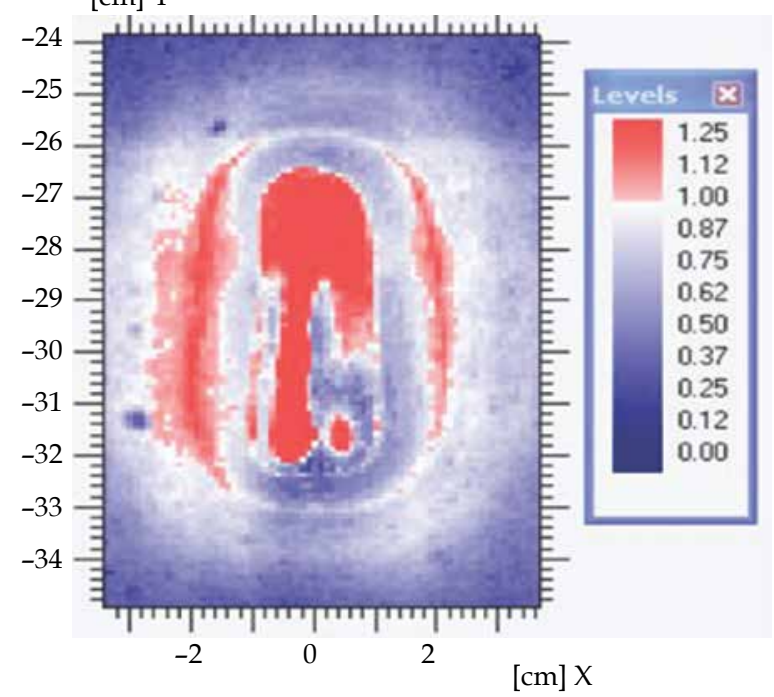

$[\mathrm{cm}] \mathrm{Y}$

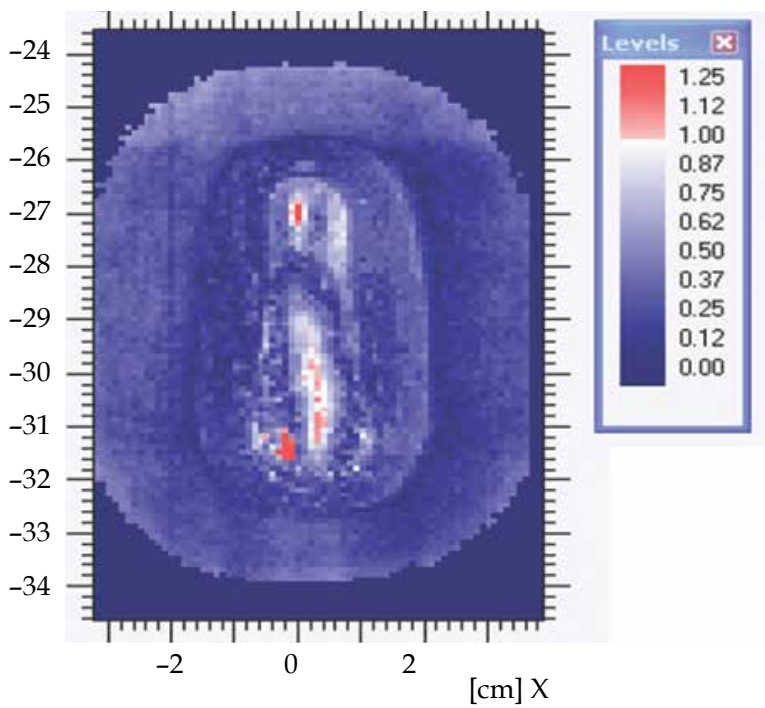

Fig. 4. 2D dose profiles comparison showing the calculated dose (green) and the measured (red) dose and 2-d planar dose differences for A) water-filled balloon, TG-43 calculation; B) air-filled balloon, TG-43 calculation; C) air-filled balloon, TG-186 calculation 
For the single central catheter, the dose at the applicator surface was approximately $170 \%$, at $5 \mathrm{~mm}, 100 \%$, and at $10 \mathrm{~mm}$ from the surface, it was $71 \%$. For the 3 -catheter example, these values were $200 \%, 100 \%$, and $33 \%$, and the contralateral surface was $60 \%$. For the 6-catheter case, the corresponding doses were $200 \%, 100 \%$, and $50 \%$.

\section{Discussion}

We investigated the effect of the presence of air within the therapeutic balloon compared to water on the dose distribution. This has been discussed with recommendations published [17] in regard to brachytherapy. Our data support these recommendations in that measured dose distributions agree very well with model-based dose calculations. The effect of going from TG-43 to TG-186 was modest. Implant $\mathrm{V}_{100}$ and $\mathrm{V}_{150}$ values changed about $2 \%$ in the case of using all 6 channels. In the 3-channel case, the changes were slightly larger ( $3 \%$ to $5 \%$ ). This is likely due to the fact that full scatter was provided on the tissue side of the applicator.

Similar results have been reported by others [18]. In a retrospective dosimetric comparison for 38 accelerated partial breast irradiation patients [19], small but significant changes in clinically relevant DVH parameters were seen when TG-186 calculations were used in place of TG-43 on Oncentra planning platform. In another recent study [20], it was suggested that ACE may underestimate dose to bone by as much as $10 \%$, and that TG- 43 might be better for dose calculations in scalp brachytherapy. It appears that the clinical usefulness and significance of TG-186 calculation should be evaluated for specific sites and applicators.

The purpose for the design of this applicator was to provide asymmetry in the dose distribution, so as to be able to conform dose coverage to targets that vary in size and position. Clearly, a single-catheter only provides a symmetric distribution in the radial direction, therefore, may overdose some parts of the esophagus in order to cover the greatest extent of the disease. Single-catheter applicators, unless equipped with centering devices, may change position radially resulting in overdosing and underdosing different regions. Further, even with a centering device, a single central catheter cannot modulate dose to a tumor radially, which may have different thicknesses at different locations around the diameter and along the length of the esophagus. The anchor and therapeutic balloons along with 6 peripheral channels were designed to minimize such positional effects while

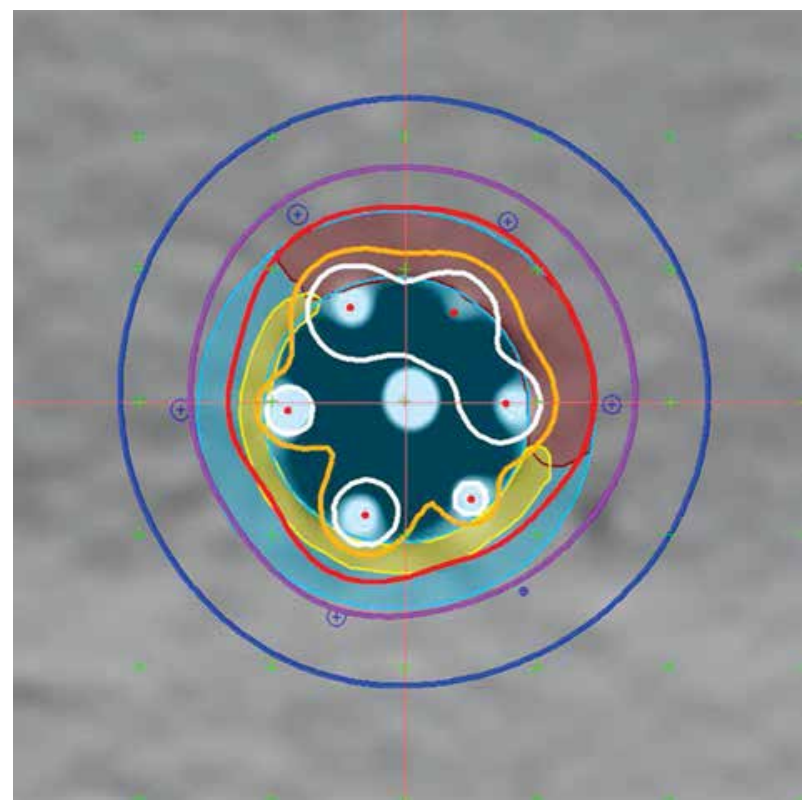

Fig. 5. Central cross-sectional planar dose distribution showing 2 targets treated with 6 catheters. Targets are yellow and brown. Isolines shown are as in Figure 2 (no 60\%)

offering the possibility of improving dose conformity to the target. Data presented in Table 1 indicate that for partial ring targets, this applicator has superior sparing of non-target tissue. Thus, for the 1 vs. 3 catheter case, the sparing was 10-3.4 - $6.6 \mathrm{cc}(66 \%)$, and for the 1 vs. 6 case, it was 6.6-1.7 - 4.9 cc (75\%). Target coverage was maintained in all cases $(99 \%)$. The conformity index also reflected better conformity to the target improvement, 2.5 vs. $1.5(40 \%)$ and 1.7 vs. $1.2(30 \%)$.

The dose estimates for the simulated single-catheter case are in agreement with published data for a commercial applicator (no longer available it seems) that provides centering balloons [21]. A multi-channel applicator for esophageal treatments has also been described [22]. It is non-inflatable, has 10 peripheral channels, and allows insertion of endoscope in central channel. The main advantage of the one described here is its inflatability to different diameters. The discussed dosimetric examples were for a $40 \mathrm{ml}$ air inflation yielding a $1 \mathrm{~cm}$ applicator radius. Greater or lesser inflations are possible. Because of the geometry of our applicator and the proximity of source dwell positions to the applicator surface, the maximum doses to surrounding tissue may be considerably higher than for a single-centered catheter applicator; although,

Table 1. Dose-volume comparison between the multi-channel and single channel applicators for 3 cases

\begin{tabular}{lccccc} 
Case & No. of catheters & Target volume $[\mathrm{cc}]$ & $\begin{array}{c}\text { Treatment } \\
\text { volume }[\mathrm{cc}]\end{array}$ & $\mathrm{Cl}$ & Ring non-target $\mathrm{V}_{100}[\mathrm{cc}]$ \\
\hline Ring & 1 & 16.6 & 16.6 & 1.0 & 0 \\
\cline { 2 - 6 } & 6 & 16.6 & 16.6 & 1.0 & 0 \\
\hline Partial ring with 1 thickness & 1 & 6.6 & 16.6 & 2.5 & 10.0 \\
\cline { 2 - 6 } & 3 & 6.6 & 10.0 & 1.5 & 3.4 \\
\hline Partial ring with 2 thicknesses & 1 & 10.0 & 16.6 & 1.7 & 6.6 \\
\cline { 2 - 6 } & 6 & 10.0 & 11.7 & 1.2 & 1.7
\end{tabular}


that may be less for a catheter without a centering device. Prescription doses would need to be reconsidered in that light. However, it should be noted that repositioning the device from one fraction to the next and the effects of breathing motion would tend to distribute hot spots, thereby, lessening the potential damage.

The concept of inducing dose asymmetry by using several peripheral channels has been applied to commercial applicators intended for other sites. For example, a multi-channel vaginal cylinder (Elekta) and an inflatable multi-channel balloon for partial breast radiotherapy (SAVI, Cianna Medical Inc., Aliso Viejo, USA) have been available for some years. An inflatable applicator for treating rectal lesions has been described recently [23]. The concept is unusual in that the catheters all seem to be placed on one side of the inflating rectal balloon and may limit the circumferential size of the intended target.

All of the above applicators offer the possibility of reducing dose to and thus, sparing the contralateral side of the treatment site. Attention to increased surface dose at the ipsilateral side is warranted.

\section{Conclusions}

A balloon inflatable, multi-channel applicator for endoluminal HDR treatment of esophageal cancer has been developed and tested in vitro. It permits several degrees of freedom in conforming the dose distribution to non-uniform, asymmetric targets.

The use of model-based dose calculations produced only a small change in the dose distribution due to the presence of a significant air volume.

\section{Acknowledgment}

This work was supported in part by a grant from the Cleveland Clinic Taussig Cancer Center's Scott Hamilton CARES initiative.

\section{Disclosure}

The authors report no conflict of interest.

\section{References}

1. Spechler S. Risk stratification for esophageal adenocarcinoma screening and surveillance. Gastro Hepat 2006; 11: 798-799.

2. Zhang Y. Epidemiology of esophageal cancer. World J Gastroenterol 2013; 19: 5598-5606.

3. Siegel R, Miller K, Jemal A. Cancer statistics, 2018. CA Cancer J Clin 2018; 68: 7-30.

4. Bray F, Ferlay J, Soerjomataram I et al. Global cancer statistics 2018: GLOBOCAN estimates of incidence and mortality worldwide for 36 cancers in 185 countries. CA Cancer J Clin 2018; 68: 394-424.

5. al-Sarraf M, Martz K, Herskovic A et al. Progress report of combined chemoradiotherapy versus radiotherapy alone in patients with esophageal cancer: an intergroup study. J Clin Oncol 1997; 15: 277-284.

6. Minsky BD, Pajak TF, Ginsberg RJ et al. INT 0123 (Radiation Therapy Oncology Group 94-05) phase III trial of combined-modality therapy for esophageal cancer: high-dose versus standard-dose radiation therapy. J Clin Oncol 2002; 20: 1167-1174.
7. Ishikawa H, Nonaka T, Sakurai H et al. Usefulness of intraluminal brachytherapy combined with external beam radiation therapy for submucosal esophageal cancer: long-term follow-up results. Int J Radiat Oncol Biol Phys 2010; 76: 452-459.

8. Wong S, Hennequin C, Quero L. Curietherapie du cancer de 1-oesophage. Cancer/Radiotherapie 2013; 17: 159-161.

9. Gaspar L, Nag S, Herskovic A et al. American Brachytherapy Society [ABS] consensus guidelines for brachytherapy of esophageal cancer. Int J Radiat Oncol Biol Phys 1997; 38: 127-132.

10. Folkert M, Cohen G, Wu A et al. Endoluminal high-dose-rate brachytherapy for early stage and recurrent esophageal cancer in medically inoperable patients. Brachytherapy 2013; 12 : $463 \mathrm{e} 470$.

11. Gaspar LE, Winter K, Kocha WI et al. A phase I/II study of external beam radiation, brachytherapy, and concurrent chemotherapy for patients with localized carcinoma of the esophagus (Radiation Therapy Oncology Group Study 9207): final report. Cancer 2000; 88: 988-995.

12. Tamaki $\mathrm{T}$, Ishikawa $\mathrm{H}$, Takahashi $\mathrm{T}$ et al. Comparison of efficacy and safety of low-dose-rate vs. high-dose-rate intraluminal brachytherapy boost in patients with superficial esophageal cancer. Brachytherapy 2012; 11: 130-136.

13. Lancellotta V, Cellini F, Fionda B et al. The role of interventional radiotherapy (brachytherapy) in stage I esophageal cancer: an AIRO (Italian Association of Radiotherapy and Clinical Oncology) systematic review. Eur Rev Med Pharmacol Sci 2020; 24: 7589-7597.

14. Lancellotta V, Cellini F, Fionda B et al. The role of palliative interventional radiotherapy (brachytherapy) in esophageal cancer: An AIRO (Italian Association of Radiotherapy and Clinical Oncology) systematic review focused on dysphagia-free survival. Brachytherapy 2020; 19: 104-110.

15. Greskovich J, Kolar M, Godley A et al. Improved CTV dose homogeneity and normal tissue maximum dose for esophageal HDR using a three-tube technique. Radiother Oncol 2012; 103: S157.

16. Anagnostopoulos G, Baltas D, Pantells E et al. The effect of patient inhomogeneities in oesophageal 192Ir HDR brachytherapy: a Monte Carlo and analytical dosimetry study. Phys Med Biol 2004: 49: 2675-2685.

17. Beaulieu L, Tedren AC, Carrier JF et al. Report of the Task Group 186 on model-based dose calculation methods in brachytherapy beyond the TG-43 formalism: current status and recommendations for clinical implementation. Med Phys 2012; 39: 6208-6236.

18. Ma Y, Vilande J, Ballester F et al. A generic TG-186 shielded applicator for commissioning model-based dose calculation algorithms for high-dose-rate 192 Ir brachytherapy. Med Phys 2017; 44: 5961-5976.

19. Zourari K, Major T, Herein A et al. A retrospective dosimetric omparison of TG43 and a commercially available MBDCA for an APBI brachytherapy patient cohort. Phys Med 2015; 31 : 669-676.

20. Cawston-Grant B, Morrison H, Sloboda R et al. Experimental assessment of the Advanced Collapsed-cone Engine for scalp brachytherapy treatments. Brachytherapy 2018; 17: 489-499.

21. Cohen G, Goodman K, Wu A et al. Clinical use of a novel balloon based esophageal brachytherapy applicator. Brachytherapy 2016; 15: S35.

22. Berger D, Schmid R, Wisgrill B et al. Endoscope-guidable multichannel applicator for brachytherapy of esophageal cancer. Brachytherapy 2017; 16: S85.

23. Schmid MP, Berger, D, Heilmann M et al. Inflatable multichannel rectal applicator for adaptive image-guided endoluminal high-dose-rate rectal brachytherapy: design, dosimetric characteristics, and first clinical experiences. J Contemp Brachytherapy 2017; 9: 359-363. 